\title{
Begutachtungsleitlinien Psychosomatische Medizin
}

a PD Dr. med. Niklaus Egloff; ${ }^{b}$ Prof. Dr. med. Rainer Schaefert, ${ }^{c}$ Dr. med. Pierre Loeb, ${ }^{d}$ lic. phil. Beat Steiger, e Prof. Dr. med. Roland von Känel

${ }^{a}$ Kompetenzbereich Psychosomatische Medizin, Universitätsklinik für Neurologie, Universitätsspital Inselspital, Bern; b Klinik für Psychosomatik, Bereich Medizin, Universität und Universitätsspital Basel, Basel; ' Wissenschaftlicher Beirat Schweizerische Akademie für Psychosomatische und Psychosoziale

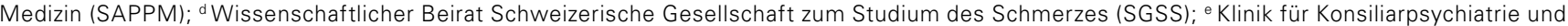
Psychosomatik, UniversitätsSpital Zürich, Zürich

Die Beurteilung von Patienten* mit psychosomatischen Syndromen stellt hohe Ansprüche an den Gutachter. Die hierfür entwickelten Begutachtungsleitlinien fokussieren auf positive Hinweiskriterien. Damit werden die Leitlinien der neuen bundesrichterlichen Rechtspraxis gerecht, welche die Formulierung von «Indikatoren» für die Beschreibung der Gesundheitsstörungen und Beeinträchtigungen fordert. Entsprechend der Ständeratsdebatte vom 14. September 2017 propagieren die Leitlinien explizit auch die verstärkte Integration der hausärztlichen Beurteilung bei diesen Patienten. Insgesamt widerspiegelt sich in den vorgestellten Leitlinien ein sowohl juristisch wie auch medizinisch gewandeltes Verständnis psychosomatischer Erkrankungen.

\section{Paradigmenwechsel beim Schweize- rischen Bundesgericht}

Mit einem ebenso mutigen wie unerlässlichen Leiturteil vom 03.06.2015 (9C_492/2014) nahm das Bundesgericht Abstand von seiner bisherigen Praxis der Rechtsprechung zu somatoformen Schmerzstörungen [1]. Insbesondere wurde das Konstrukt der «willentlichen Überwindbarkeitsvermutung» verworfen, welches von 2004-2015 gewissermassen als Pauschalablehnungsargument von IV-Anträgen bei dieser Art von Erkran-

\section{Das Bundesgericht fordert eine «einzelfall- gerechte» und «ergebnisoffene» Beurteilung sämtlicher Erkrankungen, ungeachtet ihrer Ätiologie.}

kungen galt [2]. Stattdessen fordert das Bundesgericht neu eine "einzelfallgerechte» und "ergebnisoffene» Beurteilung sämtlicher Erkrankungen, ungeachtet ihrer Ätiologie.

Als Konsequenz dieser Revision forderte das Schweizerische Bundesgericht die zuständigen medizinischen Fachgesellschaften auf, zeitgemässe medizinische Leitlinien zur Begutachtung psychosomatischer Leiden auszuarbeiten [2]. Denn nicht zuletzt in Ermangelung entsprechender medizinischer Leitlinien war in der Schweiz zwischen 2004 und 2015 eine vorwiegend durch die Rechtsprechung definierte Begutachtungskultur dieser Erkrankungen entstanden. Diese Sachlage war für alle beteiligten Akteure nicht zufriedenstellend und löste fachliche Kritik und Irritation aus $[3,4]$.

In einem Positionspapier erklärten sich die Schweizerische Akademie für Psychosomatische und Psychosoziale Medizin (SAPPM) und die Schweizerische Gesellschaft zum Studium des Schmerzes (SGSS) 2016 bereit, entsprechende Begutachtungsleitlinien nach aktuellsten medizinischen Erkenntnissen zu erarbeiten [5]. Das zwischenzeitlich von einer interdisziplinären Expertengruppe erarbeite Leitliniendokument liegt nun seit dem 01.01.2018 vor.

\section{Leitlinien nach neuem Muster}

Es bestehen in der Schweiz diverse Vorstösse, die Qualitätssicherung der medizinischen Begutachtung

Das gesamte 20-seitige Leitliniendokument ist online unter https://www.sappm.ch/ueber-uns/begutachtung/ abrufbar. 
grundsätzlich zu verbessern [6]. Vorliegende Leitlinien sind formal-versicherungsmedizinisch kongruent mit anderen jüngst revidierten Begutachtungsleitlinien, z.B. jener der Schweizerischen Rheumatologischen Gesellschaft und jener der Schweizerischen Gesellschaft für Psychiatrie und Psychotherapie [7,8]. Inhaltlich schliessen die vorliegenden Leitlinien die bisherige Lücke zwischen den beiden genannten Leitlinien hinsichtlich des Gebiets funktioneller Körperbeschwerden. Explizit fokussieren die Leitlinien dabei auf die sehr häufige Thematik «somatoformer Schmerzerkrankungen». Was die fachliche Beurteilung von Schmerzerkrankungen anbelangt, besteht eine sehr hohe Übereinstimmung mit den kürzlich revidierten Deutschen Leitlinien "Ärztliche Begutachtung von Menschen mit chronischen Schmerzen" [9].

Das vom Bundesgericht neu geforderte strukturierte Beweisverfahren gliedert sich in eine logische Abfolge definierter Informationseinheiten (Abb.1). Zu jeder dieser Informationseinheiten bieten die Leitlinien massgebliche Indikatoren zur Evaluation des Einzelfalls. Die einzelnen Informationseinheiten bilden gemeinsam eine Argumentationslinie, die schliesslich die gutachterliche Arbeitsunfähigkeitseinschätzung begründen soll. Ausdrücklich verabschiedete sich das
Bundesgericht 2015 von einer abhakbaren KriterienCheckliste und fordert neu explizit eine individualisierte Gesamtgewichtung von Indikatoren mit dem übergeordneten Ziel einer konsistenten und einzelfallgerechten Rechtsprechung [1].

Das Schweizerische Bundesgericht propagierte mit seinem Leiturteil $2015 \mathrm{zu}$ somatoformen Schmerzerkrankungen ein neuartig strukturiertes gutachterliches Beweisverfahren. Vorliegende Graphik (Abb. 1) zeigt die Umsetzung dieses Beweisverfahrens aus fachmedizinischer Sicht durch eine logische Abfolge definierter Informationseinheiten; diese bilden gemeinsam eine Argumentationslinie, aus der sich schliesslich die gutachterliche Einschätzung begründet.

\section{Abschied von einem veralteten dualis- tischen Krankheitsverständnis}

Bis vor kurzem basierte die Rechtsprechung auf einem einseitig organzentrierten Medizinalverständnis, welches als «Beweis» für ein geklagtes Symptom einen «objektivierbaren" strukturellen Organbefund forderte. Bei klassischen Organkrankheiten mag diese Art von Objektivierungsmethode weitgehend sinnvoll sein. Bei psychosomatischen respektive funktionellen

Ausprägung und Art der hauptdiagnoserelevanten Symptome und Befunde (Akten, Anamnese, Status, Psychometrie)

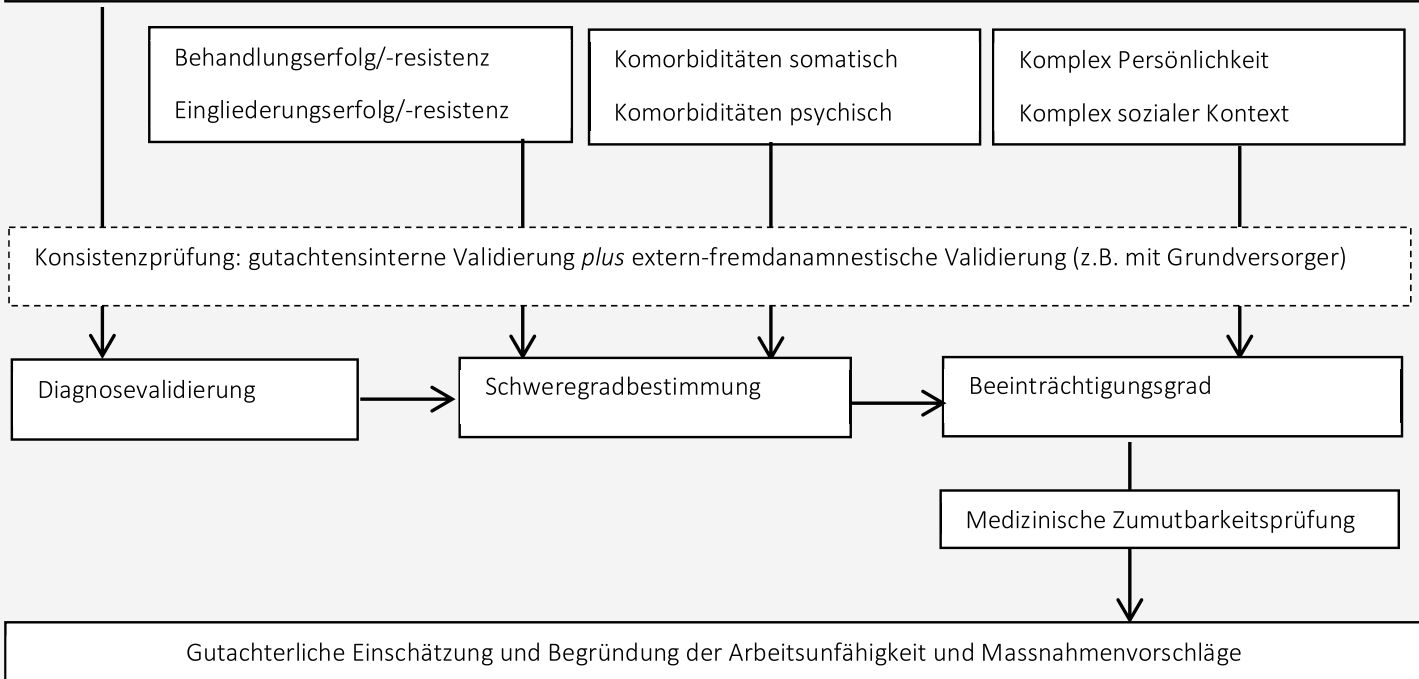

Abb. 1: Übersicht Gutachtensablauf: Das Schweizerische Bundesgericht propagierte mit seinem Leiturteil 2015 zu somatoformen Schmerzerkrankungen ein neuartig strukturiertes gutachterliches Beweisverfahren, welches «einzelfallgerecht» und «ergebnisoffen» sämtliche Krankheitsfaktoren und Beeinträchtigungen ungeachtet ihrer Ätiologie beurteilen soll. Die vorliegende Graphik zeigt die Umsetzung dieses Beweisverfahrens aus fachmedizinischer Sicht durch eine logische Abfolge definierter Informationseinheiten; diese bilden gemeinsam eine Argumentationslinie, aus der sich schliesslich die gutachterliche Einschätzung begründet. 
Krankheiten ist dieses Nachweisverfahren aber zum Scheitern verurteilt, da hier serologisch, zytologisch, histologisch oder radiologisch keine nachweisbaren Veränderungen vorliegen müssen.

Gemäss einer veralteten, seit Descartes bestehenden, dualistischen Ausschlusslogik erforderte das «Fehlen» eines klassisch-organischen Befundes zumindest das Vorhandensein einer erheblichen psychischen Störung. Entsprechend wurden bei Patienten mit funktionellen Körperbeschwerden regelhaft psychiatrische

\section{In einem Positionspapier erklärten sich die SAPPM und die SGSS bereit, entsprechende Begutachtungsleitlinien nach aktuellsten medizinischen Erkenntnissen zu erarbeiten}

Teilgutachten durchgeführt, welche zwar oft leichte bis mittelgradige depressive Begleiterkrankungen beschrieben, indessen damit keine genügende Informationslage bezüglich der geklagten körperlichen Leistungseinschränkungen lieferten. Das heisst, mit diesem obsoleten dualistischen Krankheitsverständnis fielen Patienten mit psychosomatischen Körpersymptomstörungen regelhaft bei der Begutachtung zwischen Stuhl und Bank.

\section{Modernes Krankheitsverständnis und gutachterliche Diagnosesicherung}

Mit der Zunahme stressassoziierter Gesundheitsprobleme hat sich die Wahrnehmung von psychosomatischen Erkrankungen in den letzten 20 Jahren stark gewandelt. Auf Basis neurowissenschaftlicher und psychobiologischer Erkenntnisse hat sich ein integratives Krankheitsverständnis von psychosomatischen Erkrankungen in der Medizin entwickelt [10,11]. Die Pathophysiologie dieser Erkrankungen ist komplex und vielschichtig. Nach heutigem Wissen handelt es sich um körperliche Symptome, die sich im Rahmen von übergeordneten Perzeptions-, Regulations- und Prägungsprozessen des Zentralnervensystems erklären lassen. Das Störungsniveau liegt dabei wesentlich in der Interaktion des Zentralnervensystems mit peripheren Organen. Diese Interaktion unterliegt einem neuroendokrinen, immunologischen und autonomen Wechselspiel, welches massgeblich durch das psychische Erleben beeinflusst wird [12].

Moderne Begutachtungsleitlinien der Psychosomatischen Medizin müssen der Vielschichtigkeit der Pathogenese dieser Erkrankungen gerecht werden. Entsprechend erfordert der Abschnitt Diagnosesicherung im Gutachtensablauf die Überprüfung von Indikatoren auf allen genannten Niveaus: Geprüft werden Hinweise veränderter Körperperzeption, vegetativer Dysregulation, neuropsychischer Stresssymptome, psychischer Symptome, veränderter Verarbeitungs- und Verhaltensmuster sowie des biographischen Prägungsprofils. Psychische Komorbiditäten kommen gehäuft bei psychosomatischen Körpersymptomstörungen vor. Diese sind aber weder Krankheitsvoraussetzung noch primäre Entstehungsursache der Körperfunktionsstörungen, sondern oftmals genauso Folge derselben anhaltenden psychobiographischen Stressbelastungen wie die Körpersymptomstörung selbst. Die Lebensgeschichte und das Verhalten der Patienten zeigen entsprechend regelhaft Merkmale und Muster einer ungünstigen stressbiographischen Prägung, welche die psychischen und psychosomatischen Beschwerden gleichermassen plausibilisieren [13,14].

\section{Konsistenzprüfung unter Beiziehen des Hausarztes}

Für die externe Konsistenzprüfung muss beurteilt werden, ob sich das erfasste Leiden auch mit fremdanamnestisch-externen Angaben deckt. Hierfür kommt neben Eindrücken aus einer allfälligen Psychotherapie oder aus Eingliederungsmassnahmen vor allem der hausärztlichen Langzeitbeobachtung eine wichtige Bedeutung zu. Der Hausarzt hat oftmals den umfassendsten und differenziertesten Einblick, inwiefern sich die beklagten Funktions- und Leistungseinschränkungen in den Bereichen Arbeit, Haushaltführung und sozialen Beziehungen / Freizeit bei längsschnittlicher Betrachtung analog darstellen. Eine gute hausärztliche Dokumentation dieser Aspekte ist daher sehr hilfreich. Der vorliegende Leitfaden Psychosomatische Medizin betont die Wichtigkeit der Rolle des Hausarztes entsprechend der Gutachtens-Diskussion im Rahmen der Ständeratsdebatte vom 14. September 2017 [6].

Im Berufsalltag der Betroffenen führen konkret oftmals die folgenden Aspekte zu Funktionsbeeinträchtigungen:

Das vom Bundesgericht neu geforderte strukturierte Beweisverfahren gliedert sich in eine logische Abfolge definierter Informationseinheiten.

Stressassoziierte Symptomverstärkung (z.B. Schmerzverstärkung in Bezug auf das geforderte Arbeitstempo, den Verantwortungsdruck und das Arbeitspensum); reduzierter mentaler Belastungsgrad in Bezug auf die Tätigkeitsanforderungen und Sicherheitsansprüche des Betriebs (Konzentrationsfähigkeit, Gedächtnis); 
mangelnde Möglichkeit des Pacings von Arbeitseinheiten bei symptombedingt reduzierter Leistungsspanne; Überforderung hinsichtlich Anpassungsfähigkeit und sozialer Partizipation bei massgeblicher psychischer Komorbidität.

\section{Ausblick}

Mit seinem Leitentscheid vom 03.06.2015 korrigierte das Schweizerische Bundesgericht die bisherige Rechtsprechung im Bereich somatoform-funktioneller Schmerzerkrankungen und verwandter psychosomatischer Leiden [1]. Damit sollten Voraussetzungen geschaffen werden für eine Gutachtenspraxis, welche für jede Art von Erkrankung die gleichen rechtlichen Verhältnisse schafft. Die Implementierung der Begutach-

\section{Moderne Begutachtungsleitlinien der Psycho- somatischen Medizin müssen der Vielschich- tigkeit der Pathogenese dieser Erkrankungen gerecht werden.}

tungsleitlinien und deren sachgemässe Anwendung durch die beauftragten Begutachtungsinstitutionen sind die nächsten erforderlichen Schritte. Die Handhabbarkeit und Zweckmässigkeit der Leitlinien, ihre fachgerechte Anwendung und die Frage, inwiefern sich die Rechtspraxis in der Schweiz dadurch qualitativ verändert, sollte im Rahmen von wissenschaftlichen Studien evaluiert werden [15]. Aus fachmedizinischer Sicht erwarten die SAPPM und die SGSS, die Voraussetzungen für eine einzelfallgerechtere und differenzierte Beurteilung dieser Erkrankungen geschaffen zu haben.
Literatur

1 Schweizerisches Bundesgericht Urteil vom 03.06.2015 (9C_492/2014).

2 Josi P. Psychosomatische Leiden und IV-Rente: Bundesgericht ändert Rechtsprechung. Medienmitteilung des Bundesgerichtes vom 17.06.2015. https://www.bger.ch/files/live/sites/bger/files/pdf/de/9C 492_2014_2015_06_17_T_d_10_13_40.pdf

3 Henningsen P. Probleme und offene Fragen in der Beurteilung der Erwerbsfähigkeit bei Probanden mit funktionellen Körperbeschwerden. Schweizerische Zeitschrift für Sozialversicherung und berufliche Vorsorge SZS 2014;6:499-547.

4 Schweizer Radio und Fernsehen SRF. Rente weg: IV macht Kranke per Gesetz gesund. Sendung vom 29.10.2013.

5 Minzer A et al. Öffentlicher Brief an das Schweizerische Bundesgericht und das Bundesamt für Sozialversicherungen. SAEZ 2016;97(11):416-7.

6 Interpellation Graber Konrad / Berset Alain. Amtliches Bulletin 17.3366 i.R. der Ständeratsdebatte vom 14.09.2017. https://www. parlament.ch/de/ratsbetrieb/amtliches-bulletin/amtlichesbulletin-die-verhandlungen?SubjectId $=40923$

7 Leitlinien für die rheumatologische Begutachtung der Schweizerischen Gesellschaft für Rheumatologie (07/2016) www.rheuma-net.ch

8 Ebner G, Colomb E, Mager R, Marelli R, Rota F. Qualitätsleitlinien für versicherungspsychiatrische Gutachten der Schweizerischen Gesellschaft für Psychiatrie und Psychotherapie (SGPP). 3.vollständig überarbeitete und ergänzte Auflage, 2016

9 AWMF-Leitlinie für die ärztliche Begutachtung von Menschen mit chronischen Schmerzen (Leitlinie Schmerzbegutachtung), 4. Aktualisierung, 2017, AWMF-Registernummer 094-003 http:// www.awmf.org

10 Lane RD, Waldstein SR, Chesney MA, Jennings JR, Lovallo WR, Kozel PJ, Rose RM, Drossman DA, Schneiderman N, Thayer JF, Cameron OG. The rebirth of neuroscience in psychosomatic medicine, Part I: historical context, methods, and relevant basic science. Psychosom Med. 2009;71(2):117-34.

11 Lane RD, Waldstein SR, Critchley HD, Derbyshire SW, Drossman DA, Wager TD, Schneiderman N, Chesney MA, Jennings JR, Lovallo WR, Rose RM, Thayer JF, Cameron OG. The rebirth of neuroscience in psychosomatic medicine, Part II: clinical applications and implications for research. Psychosom Med. 2009;71(2):135-51.

12 Egloff N, Dungl C, Ott R. Update somatoforme Schmerzstörungen. Therapeutische Umschau 2017;74(5):254-60.

13 Egle UT, Keller F, Kappis B, Schairer U, Bär KJ. Stressinduzierte Hyper algesie (SIH). Neurobiologische Mechanismen und ihre Konsequenzen für die sozialmedizinische Begutachtung chronisch Schmerzkranker. Der Medizinisch Sachverständige 2016;112(2):73-80.

14 Landa A, Peterson BS, Fallon BA. Somatoform pain: a developmental theory and translational research review. Psychosom Med. 2012;74(7):717-27.

15 Barth J, de Boer WE, Busse JW, Hoving JL, Kedzia S, Couban R, Fischer $\mathrm{K}$, von Allmen DY, Spanjer J, Kunz R. Inter-rater agreement in evaluation of disability: systematic review of reproducibility studies. BMJ 2017;25:356;j14 